\title{
Treatment Option for Prosthetic Graft Infection after Thoracic Aorta Replacement
}

\section{Tamaki Takano*}

Department of Cardiovascular Surgery, Shinshu University School of Medicine, 3-1-1 Asahi, Matsumoto, Nagano 390-8612, Japan

Treatment of prosthetic graft infection is challenging after thoracic aorta replacement and the in-hospital mortality of graft replacement is very high at $25 \%$ to $42 \%$ [1-3]. In the treatment of prosthetic graft infections, replacement of infected graft with radical debridement has been considered ideal surgical procedures $[1,4]$. However, we may sometimes hesitate to perform prompt re-operation because of these high mortalities when the patients' activities of daily living are limited or when the patient carries the untreated infectious source of the graft infection. Medical or surgical treatment for an orthotopic infection site might be given priority over re-graft replacement because of the reinfection risk of a newly implanted graft in cases in which the persistent infection is the source of the graft infection.

One option for surgical treatment is preservation instead of removal of the prosthetic graft because removal of the graft is technically difficult and requires a long period of cardiac arrest, which may increase morbidity and mortality. The early mortality with salvage of the prosthetic graft was recently reported as $25 \%$ [3]. It is noteworthy that graft infection was successfully treated with debridement and mediastinal irrigation with povidone iodine preserving the infected graft when the patients presented with sternal wound infection. Preservation of the infected graft has been considered possible when the patients does not present with sternal wound infection before this report [5]. This paper suggests a new option for the treatment of prosthetic graft infection when the patient has re-infection risk of a newly implanted graft especially in the patient with limited activities of daily living and increased risk of mortality and morbidity.

We have recently reported the early and mid-term outcomes of prosthetic graft infection after thoracic aorta replacement [6]. The study included 8 consecutive patients with thoracic graft infection from 1997 to 2011 among 513 patients of graft replacement. We performed re-graft replacement in six patients. Of these six patients, emergency surgery was performed in two and scheduled surgery was performed in two. An unscheduled emergency surgery was required in two patients for graft detachment during the medical treatment of the infection source. Solo medical treatment was performed in two patients without abscess or pseudoaneurysm. In-hospital mortality was $25 \%$. Re-graft infection was not observed in the six patients who underwent re-graft replacement during the 1.5- to 14-year observation period.

Prompt re-replacement of the infected graft is still favorable even when the patient has an orthotopic infection source that led to the graft infection. However, debridement with mediastinal irrigation and medical treatment preserving the infected graft may be an alternative for prosthetic graft infections if the patient does not present with an abscess or pseudoaneurysm.

\section{References}

1. Coselli JS, Köksoy C, LeMaire SA, Coselli JS, Köksoy C, et al. (1999) Management of thoracic aortic graft infections. Ann Thorac Surg 67: 19901993.

2. Czerny M, von Allmen R, Opfermann P, Sodeck G, Dick F, et al. (2011) Selfmade pericardial tube graft: a new surgical concept for treatment of graft infections after thoracic and abdominal aortic procedures. Ann Thorac Surg 92: $1657-1662$

3. Akowuah E, Narayan P, Angelini G, Bryan AJ (2007) Management of prosthetic graft infection after surgery of the thoracic aorta: removal of the prosthetic graft is not necessary. J Thorac Cardiovasc Surg 134: 1051-1052.

4. Kieffer E, Sabatier J, Plissonnier D, Knosalla C (2001) Prosthetic graft infection after descending thoracic/thoracoabdominal aortic aneurysmectomy: management with in situ arterial allografts. J Vasc Surg 33: 671-678.

5. LeMaire SA, Coselli JS (2007) Options for managing infected ascending aortic grafts. J Thorac Cardiovasc Surg 134: 839-843.

6. Takano T, Terasaki T, Wada Y, Seto T, Fukui D, et al. (2013) Treatment of Prosthetic Graft Infection after Thoracic Aorta Replacement. Ann Thorac Cardiovasc Surg.
*Corresponding author: Tamaki Takano, Department of Cardiovascular Surgery, Shinshu University School of Medicine, 3-1-1 Asahi, Matsumoto, Nagano 3908612, Japan, E-mail: ttakano@umin.ac.jp

Received July 01, 2013; Accepted July 03, 2013; Published July 05, 2013

Citation: Takano T (2013) Treatment Option for Prosthetic Graft Infection after Thoracic Aorta Replacement. J Vasc Med Surg 1: e109. doi:0.4172/2329-6925.1000e109

Copyright: ( 2013 Takano T. This is an open-access article distributed under the terms of the Creative Commons Attribution License, which permits unrestricted use, distribution, and reproduction in any medium, provided the original author and source are credited. 